\title{
Impact of overnight information on MEM volatility prediction
}

\author{
C. F. Chu And K. P. Lam
}

Overnight return in stock market is one kind of information that can reflect the volatility of the corresponding financial instrument. However, some volatility estimators, either based on range-based or high-frequency data, do not include this information in their formulations. In this study, we investigate the impact of overnight return on Engle's Multiplicative Error Model (MEM). Garman's and Hansen's whole-day-based estimators are studied to demonstrate the effects under minimum-variance situations. Besides, a general framework for incorporating overnight information is proposed and the results are discussed. Our findings demonstrate that overnight return gives a non-monotonic influence and it does contain useful information for predicting the CBOE volatility indexes under specific combinations.

Keywords AND PHRASES: Volatility forecast, Multiplicative Error Model, MEM, Overnight return.

\section{INTRODUCTION}

Overnight return, the return results from the price difference between last market close and current market open, is one kind of information that can reflect the volatility of the corresponding financial instrument. However, not all volatility estimators make use of this information in their formulation. For example, the estimators proposed by Parkinson [19], Garman \& Klass $\left(\sigma_{4}^{2}\right)$ [10], Rogers \& Satchell [21] and even the standard realized variance [1] do not incorporate overnight return whereas estimators proposed by Garman \& Klass $\left(\sigma_{6}^{2}\right)$ [10], Yang \& Zhang [22] and Hansen $\&$ Lunde [12] do. There is no consensus on which kind of estimators, with and without overnight return, can better capture the underlying volatility in stock market. Furthermore, some estimators are based on daily range quotes while some are based on high frequency quotes and this further complicates the problem about the appropriateness of incorporating overnight return in the formulation of volatility estimators.

One practical usage of volatility estimators is to set them as the information sources for volatility prediction. Popular volatility prediction models such as variants of ARCH/GARCH [4, 6, 13] and RiskMetrics [25] are shown to satisfactorily capture the dynamics of stock returns. However, most of their variants are not suitable to handle nonnegative time series as the information source. To properly

handle the non-negativity, Engle proposed to model the error in the series as the multiple of the conditional mean estimates, adopting it as the mean equation in the GARCH framework [7]. This model is known as Multiplicative Error Model (MEM) and it can provide consistent results for various distributions of error terms under its quasi maximum likelihood estimation method, making it robust to ambiguous error assumptions $[8,16]$.

Besides using econometric models to measure future volatility, there is another way to indicate the level of fluctuation in the future. The Chicago Board of Options Exchange (CBOE), the world's largest options exchange, has compiled volatility indexes by averaging the weighted prices of put and call options to measure the market expectation on future volatility [24]. The CBOE market-based volatility index has been related to the model-based conditional volatility in recent literature. Blair et al. investigated the information content of VIX for the prediction of GARCH volatility and found out the current VIX value contains the richest information content for 1-step-ahead predicted realized volatility [3]. Besides, Engle \& Gallo studied the possibility of using MEM volatilities to improve the prediction of VIX in 2006. They demonstrated that multi-step average volatilities can be incorporated as statistically significant regressors in the auto-regression of VIX [8].

In this study, the impact of overnight information on volatility prediction is explored. We investigate the characteristics of MEM volatilities resulting from various degrees of overnight components and assess their incremental information content accordingly. Our study aims to address the following issues: 1) To what extent overnight information affects MEM outcomes; 2) Whether range-based and high-frequency estimators behave differently; 3) Whether the inclusion of overnight information provides additional information for predicting market-based volatilities. This study is divided into two phases to tackle these issues systematically. In the first phase, the relationships among the predicted conditional volatilities from range-based and high-frequency estimators with and without overnight information are studied. In addition to the defined minimumvariance situations $[10,12]$, a generalized framework is proposed to broaden the investigation. Afterward, the incremental information content of the predicted volatilities is assessed by the improvements on the auto-regression of market-based volatility indexes in the second phase. 
This paper is organized as follows. Specifications of various volatility estimators are in Section 2. A brief introduction of Multiplicative Error Model (MEM) and the model settings for our study are in Section 3. Empirical investigations of impacts of overnight information are discussed in Section 4 and Section 5 states the conclusion.

\section{VOLATILITY ESTIMATORS}

Estimating volatility has long been a major issue in the financial literature. Classical estimation method assumes underlying stock price follows a simple Brownian motion and takes squared log daily return as an unbiased variance estimator. However, this simple estimator cannot satisfactorily capture the underlying dynamics and was shown to be not efficient [10]. In 1980, Parkinson formulated a way to use high and low price to better capture the underlying dynamics [19]. In the same year, Garman and Klass proposed a minimum-variance unbiased variance estimator for a simple Brownian motion [10]. Ten years later, an estimator for a drifted Brownian motion was proposed by Rogers and Satchell. It was a drift-independent variance estimator that was proved to be unbiased for Brownian motion with drift [21]. Besides, Yang and Zhang formulated a multipleday-averaged drift independent minimum-variance unbiased variance estimator in 2000 [22]. These volatility estimators are called range-based estimators as they are calculated by using open, high, low and close information.

Another stream of volatility estimators is called highfrequency estimators as they are based on the highfrequency intraday return information. The most wellknown estimator is the standard realized variance proposed by Andersen and Bollerslev in 1998 [1]. This estimator is formulated as the sum of squared high-frequency intraday returns. However, studies showed that this estimator becomes biased with the increase of sampling frequency [2, 11, 17]. The bias is caused by the autocorrelation effect resulting from non-synchronous trading, bid-ask spread and data extrapolation in the real market and the influence is collectively regarded as a market microstructure noise. The noise may bias the results and various ways have been proposed to tackle this problem $[2,17]$. Usually, higher sampling frequency will lead to a more significant noise problem. One practical work-around is to select a sampling frequency that is not too high to avoid the autocorrelation problem. On the contrary, range-based estimators do not suffer from the microstructure noise problem and they have been shown to be robust under a simulation experiment [23]. It is still a controversial issue to decide which type of estimators, range-based or high-frequency, can express the volatility best.

Four estimators are selected as input sources for MEM models in our study. Garman and Klass's estimators are selected as range-based estimators to measure the volatility in an active trading period $\left(\hat{\sigma}_{4}^{2}\right)$ and a whole day period $\left(\hat{\sigma}_{6}^{2}\right)$

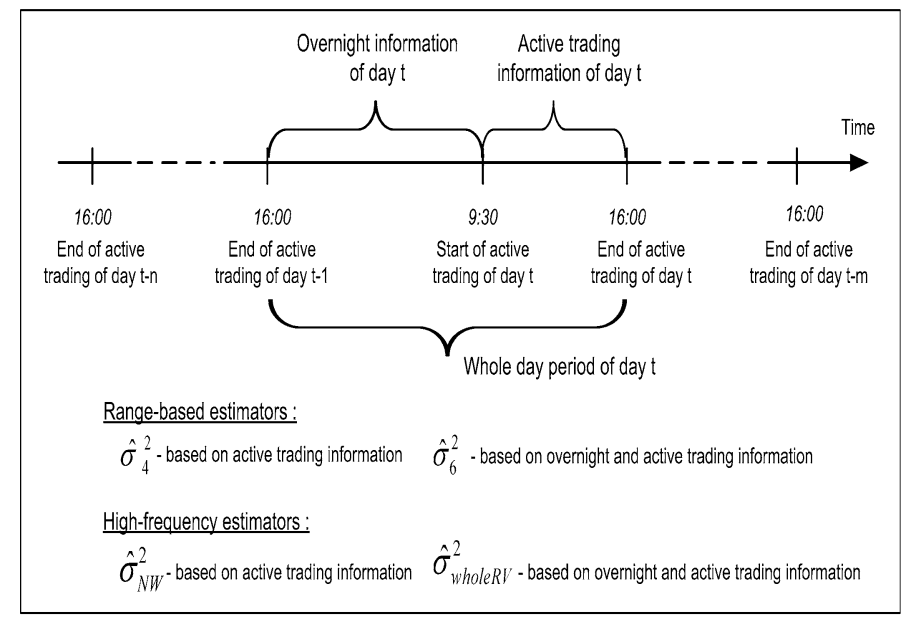

Figure 1. Illustration of the scope of four estimators.

respectively. For high frequency estimators, Newey-West realized variance $\left(\hat{\sigma}_{N W}^{2}\right)$ and Hansen and Lunde's whole-daybased minimum-variance realized variance $\left(\hat{\sigma}_{\text {wholeRV }}^{2}\right)$ are chosen. Figure 1 illustrates the time spans of these estimators. Overnight information is embedded in both whole-daybased estimators, $\hat{\sigma}_{6}^{2}$ and $\hat{\sigma}_{w h o l e R V}^{2}$.

\subsection{Garman \& Klass's volatility estimators}

Garman and Klass proposed a number of estimators in their seminal paper in 1980 [10]. Among the few estimators, there is one for estimating the volatility in an active trading period and one for a whole day period. The estimator for an active trading period is formulated as follows.

(1) $\hat{\sigma}_{4}^{2}=0.511(u-d)^{2}-0.019[c(u+d)-2 u d]-0.383 c^{2}$

where $C_{t}=\log$ of the closing price of day $t$

$O_{t}=\log$ of the opening price of day $t$

$H_{t}=\log$ of the highest price of day $t$

$L_{t}=\log$ of the lowest price of day $t$

$u=H_{t}-O_{t}$ (normalized high)

$d=L_{t}-O_{t}$ (normalized low)

$c=C_{t}-O_{t}$ (normalized close).

The whole day version is formulated as:

$$
\hat{\sigma}_{6}^{2}=\frac{a}{f}\left(O_{t}-C_{t-1}\right)^{2}+\frac{1-a}{1-f} \hat{\sigma}_{4}^{2}
$$

where $f$ is the fraction of that day that trading is closed and it is set to $1050 / 1440$ in our study. $a$ is a weight parameter and it is set to 0.12 to achieve minimum-variance property regardless of the value of $f$.

\subsection{Realized variance and Hansen \& Lunde's whole-day-based variance estimator}

Standard Realized variance is the most well-known high frequency estimator to measure the volatility in an active 
trading period. Its formulation for day $t$ is:

$$
\hat{\sigma}_{R V}^{2}=\sum_{i=1}^{m}\left\{p\left(x_{i}\right)-p\left(x_{i}-\Delta\right)\right\}^{2}
$$

where $x_{i}$ denotes the time, $p\left(x_{i}\right)$ is the price at time $x_{i}, \Delta$ is the sampling interval, $m$ is the total number of intraday prices recorded (excluding the opening price) in a day and $p\left(x_{0}\right)$ is the opening price of day $t$.

An important concern about the estimation of realized variance is whether the return series is autocorrelated or not [18]. The standard realized variance becomes biased when the returns are autocorrelated and the result should then be adjusted accordingly. The autocorrelation phenomenon always happens in high-frequency data and there are various ways to offset the bias. Hansen \& Lunde suggested an estimation method to handle the bias and regarded it as the Newey-West modified realized variance [11]. This method is based on Bartlett kernel and is guaranteed to be nonnegative. The Newey-West modified variance for day $t$ is defined as:

$$
\begin{aligned}
\hat{\sigma}_{N W}^{2} & =\sum_{i=1}^{m} y_{i}^{2}+2 \sum_{h=1}^{q}\left(1-\frac{h}{q+1}\right) \sum_{i=1}^{m-h} y_{i} y_{i+h} \\
y_{i} & =p\left(x_{i}\right)-p\left(x_{i}-\Delta\right)
\end{aligned}
$$

the variable $q$ represents the lag-length and it is set to $q=$ $\operatorname{ceil}\left(\frac{m w}{d}\right)$ where $w$ is the desired length of lag window and $d$ is the total length of sampling period (trading period) in minutes [11].

For the whole day period, Hansen and Lunde defined an optimally combined whole day variance estimator in 2005 [12]. It is a minimum-variance estimate constructed by a weighted squared overnight return and Newey-West modified realized variance. The following equations show the settings for Hansen and Lunde's minimum-variance wholeday-based estimator $\left(\hat{\sigma}_{w h o l e R V}^{2}\right)$.

$$
\begin{aligned}
\hat{\sigma}_{\text {whole } R V}^{2} & =\omega_{1}^{*} r_{1, t}^{2}+\omega_{2}^{*} \hat{\sigma}_{N W, t}^{2} \\
\omega_{1}^{*} & =(1-\varphi) \frac{\mu_{0}}{\mu_{1}} \\
\omega_{2}^{*} & =\varphi \frac{\mu_{0}}{\mu_{2}} \\
\varphi & =\frac{\mu_{2}^{2} \eta_{1}^{2}-\mu_{1} \mu_{2} \eta_{12}}{\mu_{2}^{2} \eta_{1}^{2}+\mu_{1}^{2} \eta_{2}^{2}-2 \mu_{1} \mu_{2} \eta_{12}}
\end{aligned}
$$

where $\omega_{1}^{*}$ and $\omega_{2}^{*}$ are the optimal weight for the min-variance estimator. $r_{1, t}^{2}$ is the squared overnight return. $\mu_{0}, \mu_{1}$ and $\mu_{2}$ are the expected value of integrated variance, overnight variance and Newey-West realized variance respectively. $\eta_{1}$, $\eta_{2}$ and $\eta_{12}$ are the variance of overnight variance and NeweyWest realized variance and their covariance respectively.

\section{MODELS SPECIFICATIONS}

\subsection{MEM specification}

The way to employ volatility estimators as input series for GARCH type models is different from those for treating returns as input series. Due to the non-negative nature of the volatility estimators, it is difficult to use traditional GARCH type models, which is based on linear formulation on a return process, to estimate the model parameters as the variance and higher moments of error distribution are unlikely to be constant [7]. Engle proposed an efficient way (MEM-GARCH) to model non-negative series in GARCH framework by treating the series as a composition of its conditional mean multiplied by a unit-mean error term. This multiplicative error structure is able to provide consistent results for error terms that belong to a family of gamma distribution as the corresponding first order optimality conditions on the log-likelihood functions is the same.

The MEM $(1,1)$ model is defined by the following two equations.

$$
\begin{array}{ll}
x_{t}=\mu_{t} \epsilon_{t} & \text { Mean eqt. } \\
\mu_{t}=\omega+\alpha x_{t-1}+\beta \mu_{t-1}+c^{\prime} z_{t-1} & \text { Variance eqt. }
\end{array}
$$

In the mean equation, $x_{t}$ is the non-negative time series, $\mu_{t}$ is the conditional mean estimates and $\epsilon_{t}$ represents a unitmean gamma-distributed i.i.d. error process. The variance equation is similar as that in the GARCH framework by replacing the error squared term with $x_{t}$ in the $\mathrm{ARCH}$ term. Furthermore, exogenous variables are treated by including $z_{t}$ in the variance equation.

With the restriction of a unit-mean on the distribution of $\epsilon_{t}$, the corresponding log-likelihood function for the model is defined as $L(\theta)$.

$$
L(\theta)=\text { constant }-a \sum_{t=1}^{T}\left[-\log \left(\mu_{t}(\theta)\right)-\frac{x_{t}}{\mu_{t}(\theta)}\right]
$$

where $a$ controls the shape of the gamma distribution, $\theta$ is the parameter set $\left\{\alpha, \beta\right.$ and $\left.c^{\prime}\right\}$ to be estimated and $T$ is the size of the training sample. The first order optimality condition for maximum likelihood estimation is:

$$
\sum_{t=1}^{T}\left[-\log \left(\mu_{t}(\theta)\right)-\frac{x_{t}}{\mu_{t}(\theta)}\right]
$$

Noticing that the shape variable $a$ does not affect the first order condition and its value is irreverent and does not have any influence on $\theta$ and their standard errors $[7,8]$.

Without the justification of the underlying error distribution, it is suggested to derive the parameters in equation 10 by traditional GARCH framework as the maximizer of equation 11, which is a quasi maximum likelihood estimator. Lee \& Hansen demonstrated the Gaussian likelihood estimation 
method for $\operatorname{GARCH}(1,1)$ can provide consistently estimated parameters for input series which is neither Gaussian nor independent [16]. Referring to Engle's procedure, the parameters can be obtained by taking the positive square root of the non-negative variable of interest $\sqrt{x_{t}}$ as the dependent variable and setting the mean value to zero with the assumption of normally distributed errors $[7,8]$.

In this paper, we employ the $\operatorname{MEM}(1,1)$ framework to predict the future volatility based on the most up-to-date volatility estimates. This framework has been successfully modeled the dynamics of non-negative volatility series, including range-based and high-frequency estimates, in some applications $[8,14,15]$ and has been extended to multivariate cases recently [5]. We treat $x_{t}$ in equations 9,10 as a proxy to represent the input series (volatility estimates) for the model.

\subsection{Correlation analysis among models with and without overnight information}

Correlation analysis on the predicted conditional volatilities from MEM with and without overnight information are used to study the overnight impact. As stated in Section 2, $\hat{\sigma}_{4}^{2}$ and $\hat{\sigma}_{N W}^{2}$ are estimators without the consideration of overnight return in their formulation while $\hat{\sigma}_{6}^{2}$ and $\hat{\sigma}_{w h o l e R V}^{2}$ are estimators with overnight return.

To model the MEM 1-step-ahead conditional variances, we leave the exogenous term in equation 10 empty and replace the $x_{t}$ in equation 9 by the estimators defined in equations $1-5$ correspondingly. Since the estimators represent variance for different time spans as stated in Figure 1, their magnitudes are naturally different. Therefore, we employ correlation coefficient as a scale-independent measure to evaluate the impact of overnight information. For instance, if all overnight returns equal to zero, the correlation will be 1 . A small correlation demonstrates a large impact from overnight returns. The predicted conditional volatilities (i.e. square root of the conditional mean estimates) are named as $M E M-\sigma_{4}, M E M-\sigma_{6}, M E M-\sigma_{N W}$, and $M E M-$ $\sigma_{w h o l e R V}$ respectively for the rest of this paper for clarity.

\subsection{Generalized whole-day-based variance formulation}

Observing that the minimum-variance estimators in equations 2 and 5 can be formulated as:

$$
\operatorname{Var}_{t}=w_{1} O n_{t}^{2}+w_{2} A c t_{t}
$$

where $\operatorname{Var}_{t}$ and $A c t_{t}$ represent variance estimates of whole day period and active trading period on day $t$ respectively. $w_{1}$ and $w_{2}$ are weight factors and $O n_{t}$ is the overnight return. $\operatorname{Var}_{t}$ can then be considered as a linear combination of squared overnight return and a variance estimate of an active trading period.

The minimum-variance estimators, $\hat{\sigma}_{6}^{2}$ and $\hat{\sigma}_{w h o l e R V}^{2}$, can be considered as a particular weight combination. Scholars may use weights other than the minimum-variance weights to construct whole-day-based variance estimators. For example, Blair [3] and Gallo [9] use weights with $w_{1}: w_{2}=$ 1 : 1 to construct their estimators for whole day period. Sometimes, people may simply set $w_{1}=0$ as it is supposed that the magnitude of overnight variance is comparatively small and cannot give significant influence on the outcomes. To broaden our investigation of impact from overnight information, we try to consider all the possible combinations of positive $w_{1}$ and $w_{2}$ and formulate the estimator for whole day period as:

$$
\begin{aligned}
\operatorname{Var}_{t} & =\left(w_{1}+w_{2}\right)\left[\frac{w_{1}}{w_{1}+w_{2}} O n_{t}^{2}+\frac{w_{2}}{w_{1}+w_{2}} A c t_{t}\right] \\
& =\left(w_{1}+w_{2}\right)\left(\lambda O n_{t}^{2}+(1-\lambda) A c t_{t}\right)
\end{aligned}
$$

where $\lambda$ is some positive scalar and $0 \leq \lambda \leq 1$. $\lambda$ can be considered as a variable that governs the composition and its value indicates the influence of overnight information on a whole-day-based estimator. The portion $\lambda O n_{t}^{2}+(1-\lambda) A c t_{t}$ represents a scaled version of Var $_{t}$.

As our MEM models do not have any exogenous variable, the scaling factor, $w_{1}+w_{2}$, only adds a constant term, $T \log \left(w_{1}+w_{2}\right)$, to the likelihood function in equation 12 . The optimal conditional variances for the original series therefore only differs with the scaled version in a factor of $w_{1}+w_{2}$. In other words, the value of conditional volatilities for the original series is equal to those from scaled series multiply $\sqrt{w_{1}+w_{2}}$. Since we use correlation to compare the overnight impact and linear regression to reflect the incremental information content of the predicted conditional volatilities, the properties of scaled form $\lambda O n_{t}^{2}+(1-\lambda) A c t_{t}$ can therefore demonstrate the corresponding properties of the original series. Our generalized whole-day-based formulations are as follows.

$$
\begin{aligned}
\text { general } \hat{\sigma}_{4, t}^{2} & =\lambda O n_{t}^{2}+(1-\lambda) \hat{\sigma}_{4, t}^{2} \\
\text { general } \hat{\sigma}_{R V, t}^{2} & =\lambda O n_{t}^{2}+(1-\lambda) \hat{\sigma}_{N W, t}^{2}
\end{aligned}
$$

where general $\hat{\sigma}_{4, t}^{2}$ and general $\hat{\sigma}_{R V, t}^{2}$ represent generalized variance estimators based on range information and highfrequency information respectively. Our refined study will use these two estimators to substitute the $x_{t}$ in equations 9 and 10 for evaluating the impact of overnight return. Their corresponding predicted conditional volatilities are labeled as MEM-general $\sigma_{4}$ and MEM-general $\sigma_{R V}$.

\subsection{Regression models for VIX/VXD prediction}

The information content of MEM predicted volatilities is assessed by their influence on the prediction of market-based volatility indexes (VIX and VXD). The indexes involved are constructed by the Chicago Board Options Exchange (CBOE), the world largest options exchange, to capture market expectation of the next 30 calendar days (22 trading 
days). They are compiled by averaging the weighted prices of put and call options in the market to reflect the option implied volatilities, which in turn, represent the market's expectation of future fluctuation and hence they are called market-based volatility [20]. VIX aims to capture the volatility of S\&P 500 index while VXD aims at Dow Jones Industrial Average (DJIA) [24]. The CBOE index has been related to the model-based predicted volatility in recent literature. Blair et al. investigated the information content of VIX for the prediction of GARCH volatility and found out the current VIX value contains the richest information content for 1-step-ahead predicted realized volatility [3]. Besides, Engle \& Gallo studied the possibility of using MEM volatilities to improve the prediction of VIX in 2006. They demonstrated that multi-step average volatilities can be incorporated as statistically significant regressors in the auto-regression of VIX [8].

Under ideal efficient market hypothesis, risk neutral assumptions and the absence of insider information leakage, the model-based predicted volatility and the market-based volatility index should contain the same information content. However, these ideal situations are not valid for the real case and the information content of the two volatilities are usually different. Furthermore, their coverage on time horizon are different. The options used to compute the indexes last for around 30 calendar days covering both trading and non-trading overnight period [24] while the model-based prediction may be based on estimators without any overnight component. As the market-based volatility indexes cover the volatility for both overnight and active trading period, it is interesting to know whether the inclusion of overnight information in model-based volatility can provide any additional predictive value. For the simplicity, the existence of incremental information content of overnight return is checked by simple linear regression models based on ordinary least squares method. The general regression model is as follows:

$$
\begin{aligned}
M I_{t}= & \kappa+\gamma M I_{t-1}+\delta \text { MemRange }_{t} \\
& +\zeta \text { MemHighFreq }_{t}
\end{aligned}
$$

$M I$ is the market based index, such as VIX and VXD. $\kappa, \gamma, \delta$ and $\zeta$ are the regression coefficients, MemRange $_{t}$ and MemHighFreq $_{t}$ are the proxies that represent the MEM predicted volatilities for day $t$ with various weight on overnight composition. The estimators in equations 1,2 and 15 are substituted as the range-based proxies $\left(\right.$ MemRange $\left._{t}\right)$ while 4, 5 and 16 are as high-frequency proxies $\left(\right.$ MemHighFreq $\left._{t}\right)$ respectively.

By placing restrictions on certain parameters, we can define four different models.
1. Base model of $\mathrm{AR}(1)$ for the prediction of market based index by setting $\delta=\zeta=0$

2. Model with the use of range-based information as an additional regressor: $\zeta=0$

3. Model with the use of high-frequency information as an additional regressor: $\delta=0$

4. Unrestricted model that incorporates both range-based and high-frequency information

The regression results are compared relatively to the base specification (i.e. simple AR(1) model). Adjusted R-square and $\mathrm{F}$ statistics are used as performance measures. Although the value of market indexes constructed by CBOE represents volatilities in an annual basis, the regression models are still valid as the model coefficients can absorb the scaling effect.

\section{EMPIRICAL INVESTIGATION OF THE IMPACT OF OVERNIGHT INFORMATION}

Two index data sets, S\&P 500 and Dow Jones Industrial Average (DJIA), in the period of 13 March 2006 to 11 January 2008 (458 trading days) are used in our study. The index values are sampled every 10 minutes starting from 9:30 to $16: 00$ inclusively. In addition, the daily close of their corresponding volatility indexes, VIX and VXD, are used for the regression analysis to investigate the information content of the predicted volatilities.

Our data selection scheme for model training is based on a rolling sample approach. For instance, if the sample size is $m$, the first sample for model training will be the 1 to $m$ data points, the second sample will be the 2 to $m+1$ data points and so on. For MEM volatility forecasts, the size of an input sample is $3 / 5$ of the total available data. The set of input samples are used to produce their corresponding 1step-ahead predicted conditional volatilities. The empirical results are summarized in the subsequent sub-sections.

\subsection{Preliminary study on the empirical data}

An important concern about the calculation of realized variance is whether the return series is autocorrelated or not. If the return series is autocorrelated, the calculated result should be adjusted to offset the bias caused by the autocorrelation. In our study, the realized variance is estimated with the use of 10 minute intraday returns. Figure 2 contains the autocorrelation plots of two return series, S\&P 500 and DJIA, with $95 \%$ confidence bounds. It indicates that the first serial correlation coefficients of both series are nonzeros and the series of DJIA has some other nonzero coefficients in lag 3, 5, 9 and 10. To tackle the autocorrelation problem, we apply the Newey-West method in equation 4 to estimate the realized variances for this study. Following Hansen \& Lunde's practice, we select a fixed value for the desired length of lag window for bias adjustment [12]. The autocorrelation plot (Figure 3) for 30 minute intraday returns shows the series do not suffer from the autocorrelation 

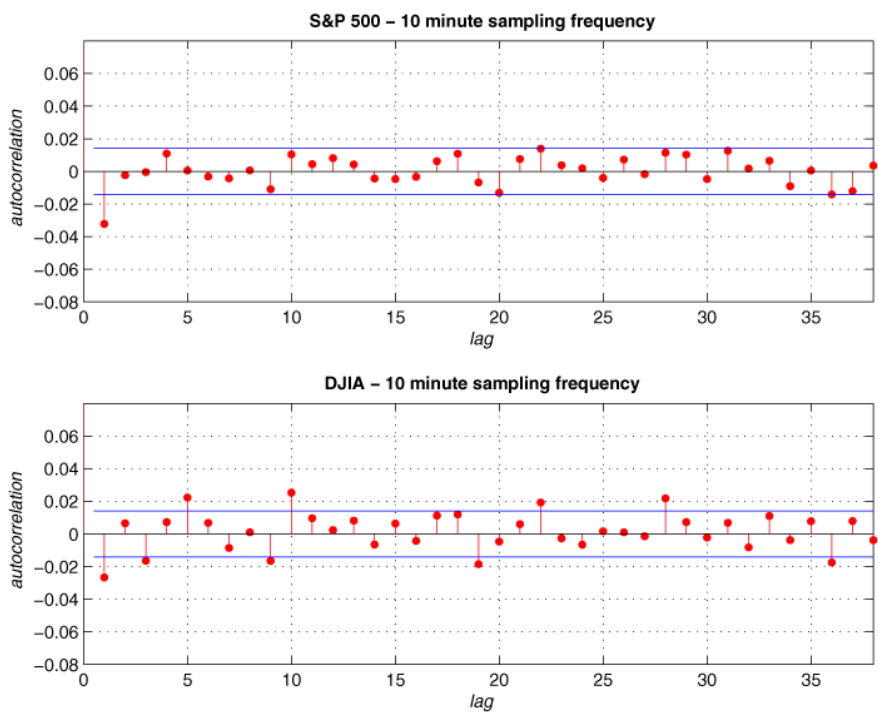

Figure 2. Autocorrelation of the 10 minute intraday NASDAQ data (with 95\% confidence level bounds).
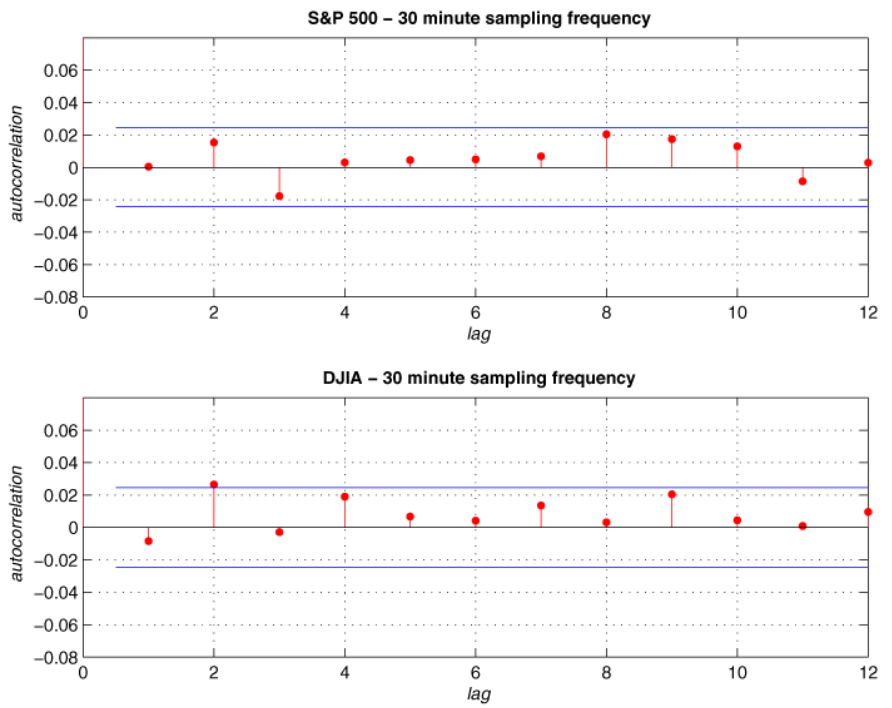

Figure 3. Autocorrelation of the 30 minute intraday NASDAQ data (with 95\% confidence level bounds).

problem and therefore we choose the length of lag window to cover 30 minutes $(w=30)$.

The descriptive statistics and correlation coefficients among squared overnight return $\left(O n^{2}\right)$, squared sigma 4 $\left(\hat{\sigma}_{4}^{2}\right)$ and Newey-West realized variance $\left(\hat{\sigma}_{N W}^{2}\right)$, are reported in Table 1.

The mean of $O n_{t}^{2}$ is much smaller and its magnitude is less than $1 / 100$ of those of $\hat{\sigma}_{4}^{2}$ or $\hat{\sigma}_{N W}^{2}$ for both S\&P 500 and DJIA cases. Furthermore, the distribution of $\mathrm{On}^{2}$ is more asymmetric and has thicker tails than the two variance series. Observations of a few large $O n_{t}^{2}$ accounts for the high
Table 1. Descriptive statistics of squared overnight return $\left(O n_{t}^{2}\right)$, squared sigma $4\left(\hat{\sigma}_{4}^{2}\right)$ and Newey-West realized variance $\left(\hat{\sigma}_{N W}^{2}\right)$

\begin{tabular}{|c|c|c|c|}
\hline \multicolumn{4}{|c|}{ S\&P 500} \\
\hline & $O n^{2}$ & $\hat{\sigma}_{4}^{2}$ & $\hat{\sigma}_{N W}^{2}$ \\
\hline Mean & $6.74 \mathrm{E}-07$ & $4.55 \mathrm{E}-05$ & $5.21 \mathrm{E}-05$ \\
\hline Median & $1.18 \mathrm{E}-09$ & $2.58 \mathrm{E}-05$ & $2.78 \mathrm{E}-05$ \\
\hline $\operatorname{Max}$ & $4.49 \mathrm{E}-05$ & $5.33 \mathrm{E}-04$ & $6.29 \mathrm{E}-04$ \\
\hline Min & 0 & $2.00 \mathrm{E}-06$ & $3.13 \mathrm{E}-06$ \\
\hline S.D. & $3.49 \mathrm{E}-06$ & $5.97 \mathrm{E}-05$ & $6.54 \mathrm{E}-05$ \\
\hline Skewness & 9.07 & 3.86 & 3.31 \\
\hline Kurtosis & 97.65 & 23.28 & 19.85 \\
\hline \multicolumn{4}{|c|}{ Correlation } \\
\hline with $O n^{2}$ & & 0.245 & 0.207 \\
\hline with $\hat{\sigma}_{4}^{2}$ & & & 0.847 \\
\hline \multicolumn{4}{|c|}{ DJIA } \\
\hline & $O n^{2}$ & $\hat{\sigma}_{4}^{2}$ & $\hat{\sigma}_{N W}^{2}$ \\
\hline Mean & $7.69 \mathrm{E}-07$ & $1.31 \mathrm{E}-04$ & $5.23 \mathrm{E}-05$ \\
\hline Median & $9.20 \mathrm{E}-09$ & $1.04 \mathrm{E}-04$ & $3.00 \mathrm{E}-05$ \\
\hline Max & $5.75 \mathrm{E}-05$ & $9.09 \mathrm{E}-04$ & $5.45 \mathrm{E}-04$ \\
\hline Min & 0 & $4.90 \mathrm{E}-06$ & $2.79 \mathrm{E}-06$ \\
\hline S.D. & $3.86 \mathrm{E}-06$ & $9.96 \mathrm{E}-05$ & $6.10 \mathrm{E}-05$ \\
\hline Skewness & 9.24 & 2.86 & 3.10 \\
\hline Kurtosis & 115.10 & 15.69 & 16.99 \\
\hline \multicolumn{4}{|c|}{ Correlation } \\
\hline with $O n^{2}$ & & -0.147 & -0.009 \\
\hline with $\hat{\sigma}_{4}^{2}$ & & & 0.794 \\
\hline
\end{tabular}

values of kurtosis. The low correlations of $O n^{2}$ to either $\hat{\sigma}_{4}^{2}$ or $\hat{\sigma}_{N W}^{2}$ indicate that it contains information other than those in the two variance series. Besides, the correlation between $\hat{\sigma}_{4}^{2}$ and $\hat{\sigma}_{N W}^{2}$ is not very high (less than 0.8) and this indicates the two estimated variance series are different from each other.

\subsection{Forecast from models with and without overnight information under minimum-variance assumptions}

As mentioned in Section 3, the estimators $\hat{\sigma}_{6}^{2}$ and $\hat{\sigma}_{\text {wholeRV }}^{2}$ consider overnight information in their formulation while $\hat{\sigma}_{4}^{2}$ and $\hat{\sigma}_{N W}^{2}$ do not. It is interesting to know whether the inclusion of overnight information in the estimators gives noticeable impacts on the predicted volatilities. MEM volatilities without overnight information (MEM- $\sigma_{4}$, MEM- $\left.\sigma_{N W}\right)$ are considered as the corresponding base series for comparison purpose. The correlation $(\rho)$ between the base series and the testing series, which contains overnight information, are calculated to reflect the impact. The value of $\rho$ will be 1 if the overnight returns are zeros and as a result, a large correlation demonstrates a small impact.

The model fitness is checked by ARCH test on the standardized residuals and Ljung-Box test on the squared standardized residuals. Since a rolling sample approach is used in this study, there are 184 MEM structures for a single 
Table 2. Diagnostics information for the MEM models

\begin{tabular}{|c|c|c|}
\hline \multirow[b]{2}{*}{ S\&P 500} & $\overline{\mathrm{ARCH}}(2)$ & $\mathrm{Q}(12)$ \\
\hline & mean & mean \\
\hline MEM- $\sigma_{4}$ & 0.476 & 8.529 \\
\hline $\mathrm{MEM}-\sigma_{6}$ & 0.476 & 8.545 \\
\hline MEM- $\sigma_{N W}$ & 0.885 & 7.139 \\
\hline MEM- $\sigma_{w h o l e R V}$ & 0.885 & 7.139 \\
\hline \multirow[b]{2}{*}{ DJIA } & $\operatorname{ARCH}(2)$ & $\mathrm{Q}(12)$ \\
\hline & mean & mean \\
\hline MEM- $\sigma_{4}$ & 0.368 & 13.415 \\
\hline $\mathrm{MEM}-\sigma_{6}$ & 0.368 & 13.439 \\
\hline $\mathrm{MEM}-\sigma_{N W}$ & 0.994 & 7.095 \\
\hline MEM- $\sigma_{w h o l e} R V$ & 0.994 & 7.095 \\
\hline
\end{tabular}

input estimator and we propose to access them by the average values of the test statistics to avoid the abnormality caused by few specific samples with highly fluctuating values. The results of $\mathrm{ARCH}(2)$ test $(5 \%$ critical value $=5.99)$ and $\mathrm{Q}(12)$ (5\% critical value $=21.03)$, are reported in Table 2 and no major specification problems are signaled by the diagnostics.

The impact of overnight information is assessed by the correlation coefficient between models with and without overnight variance. For range-based estimators, the correlation coefficients between MEM- $\sigma_{4}$ and MEM- $\sigma_{6}$ are 1.000 for both S\&P 500 and DJIA. It can be explained by the highly contrasting weight ratio in the formulation $\hat{\sigma}_{6}^{2}$. Under the minimum-variance situation, $\hat{\sigma}_{6}^{2}$ is calculated as $0.165 * O n_{t}^{2}+3.249 * \hat{\sigma}_{4}^{2}$ and the ratios of the mean of $O n^{2}$ to $\hat{\sigma}_{6}^{2}$ are $1: 68$ for S\&P 500 and $1: 170$ for DJIA. As a result, including $O n^{2}$ in the formulation gives extremely small impact to $\hat{\sigma}_{6}^{2}$ and thus the MEM- $\sigma_{6}$ should be highly correlated to MEM- $\sigma_{4}$. On the other hand, for both S\&P 500 and DJIA dataset, the correlation coefficients between MEM- $\sigma_{N W}$ and MEM- $\sigma_{\text {whole } R V}$ are also equal to 1.000 . The weight ratios between the mean of $O n^{2}$ to $\hat{\sigma}_{N W}^{2}$ are 1:77 and 1:68 for S\&P 500 and DJIA respectively and these results are different from Hansen \& Lunde's finding [12] as the magnitude of overnight variance of market index is much smaller than those appeared in individual listed stocks. With such high correlation among the MEM volatilities, it is concluded that the effect of overnight information in either Garman's or Hansen's whole-day-based variance is minimal under the minimum-variance situation.

\subsection{Impact of overnight return under a generalized weighted approach}

The empirical results in the previous section show that overnight return square $\left(\mathrm{On}^{2}\right)$ cannot give a significant impact on the MEM. However, the composition of the involved estimators, $\hat{\sigma}_{6}^{2}$ and $\hat{\sigma}_{w h o l e R V}^{2}$, are constrained by the minimum variance situation and therefore other possible combinations have not yet been studied. The minimum variance
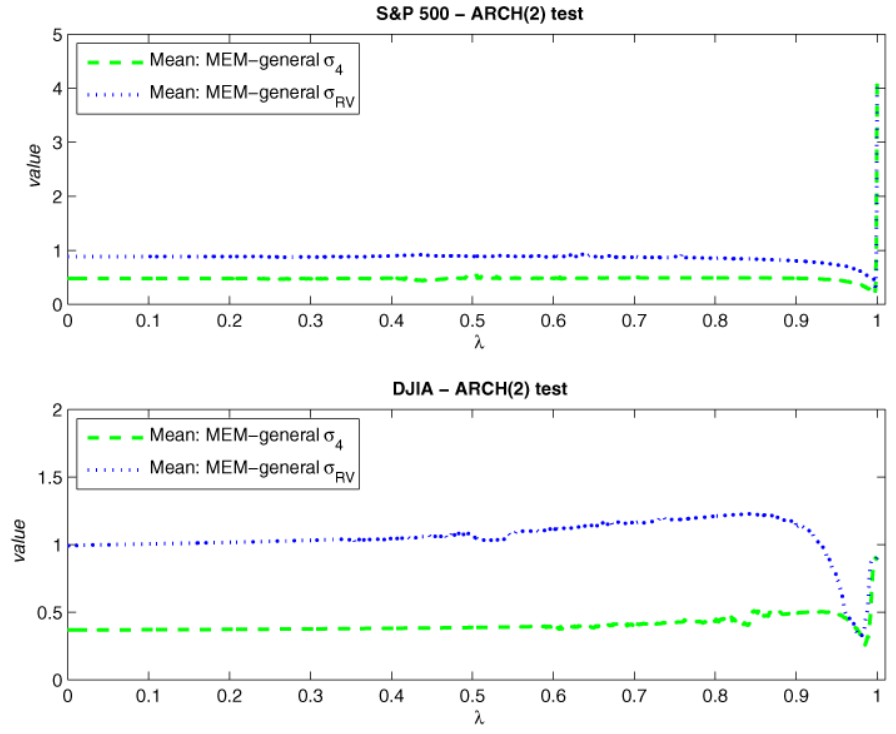

Figure 4. $A R C H(2)$ model fit measure under the generalized weighted approach.
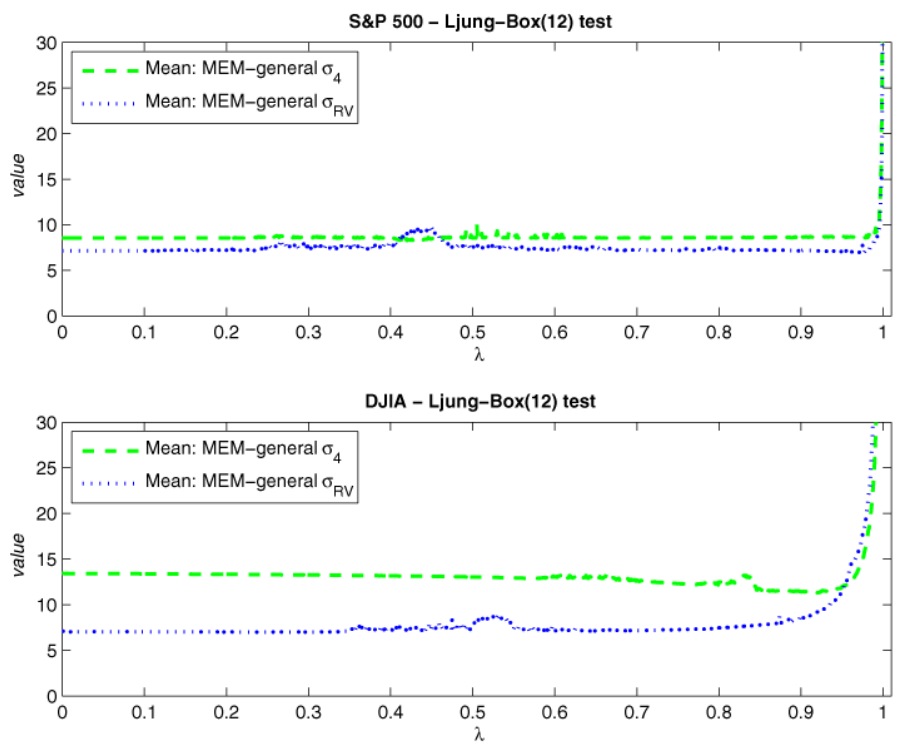

Figure 5. Ljung-Box(12) model fit measure under the generalized weighted approach.

assumption is relaxed and an investigation under the general situation specified in equations 15 and 16 is carried out. An experiment has been conducted by adjusting $\lambda$ from 0 to 1 with 0.001 for each increment and the results are recorded accordingly.

The model diagnostic information is visualized by Figures 4 and 5. In general, MEM-general $\sigma_{4}$ tends to have smaller $\operatorname{ARCH}(2)$ values, which can be interpreted as a better model fit, than MEM-general $\sigma_{R V}$ over a large range of $\lambda$. However, Ljung-Box test gives an opposite result indicat- 

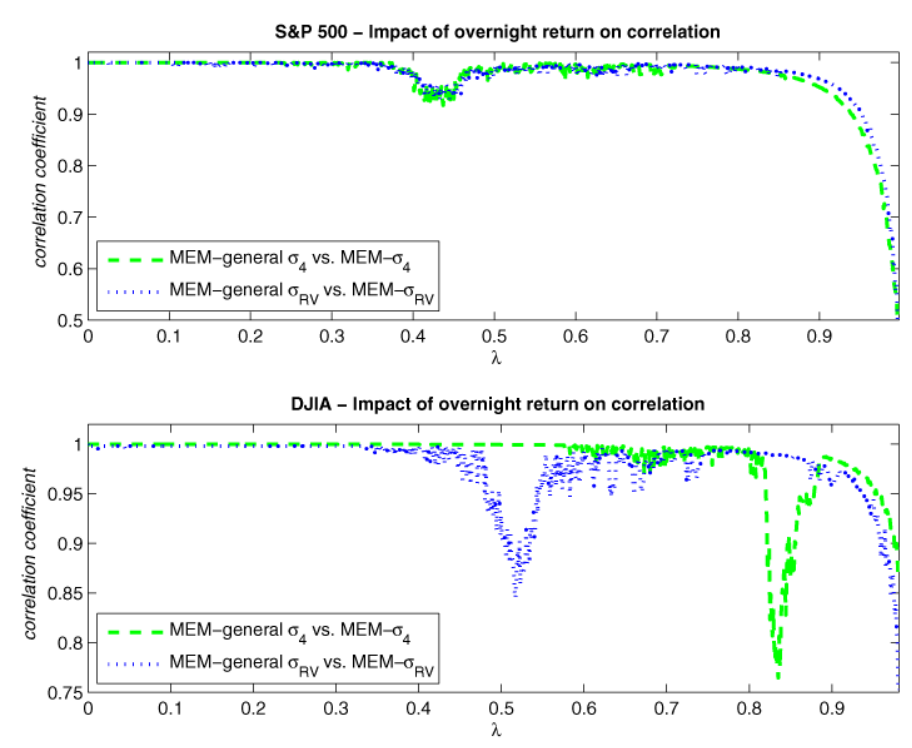

Figure 6. Comparison of correlation coefficient under the generalized weighted approach.

ing MEM-general $\sigma_{R V}$ always has a better fit. So, there is no consensus to differentiate which type of estimator has a better model fit. For the validation of MEM, all the mean values of $\operatorname{ARCH}(2)$ statistics for either MEM-general $\sigma_{4}$ or MEM-general $\sigma_{R V}$ are smaller than the critical value (5.99) for all possible $\lambda$. For Ljung-Box(12) test, the means of MEM-general $\sigma_{4}$ and MEM-general $\sigma_{R V}$ is larger than the critical value when $\lambda \geq 0.999$ for S\&P dataset. For DJIA data, the no autocorrelation hypotheses are rejected when $\lambda \geq 0.987$ and $\lambda \geq 0.982$ for the cases in MEM-general $\sigma_{4}$ and MEM-general $\sigma_{R V}$ respectively. As a result, we conclude that the MEM models are valid for S\&P 500 dataset under $0 \leq \lambda \leq 0.998$ and DJIA under $0 \leq \lambda \leq 0.981$.

The impact of overnight return is reflected by the correlation graphs. Figure 6 depicts the correlation among the predicted volatilities for $\lambda$ from 0 to 1 inclusively. To improve the visibility, the plot is zoomed to give the view for $\rho \geq 0.9$ in Figure 7 .

For S\&P 500, MEM-general $\sigma_{4}$ and MEM-general $\sigma_{R V}$ give similar behaviors. The correlation coefficients stay nearly constant (almost equal to 1 ) until $\lambda$ reaches around 0.4 and decrease sharply to the local minimum. The $\rho$ then bounds back to a high value and oscillates until $\lambda$ attains a high value. Specifically, the $\rho$ of MEM-general $\sigma_{4}$ decreases sharply from around $\lambda=0.39$ to the local minimum at $\lambda=0.437$ with $\rho=0.917$. The $\rho$ increases and rebounds to the original level at $\lambda=0.51$. A similar U-shape pattern is also observed in MEM-general $\sigma_{N W}$ but the region is shifted to $0.40 \leq \lambda \leq 0.52$ with the local minimum at $\lambda=0.433$ with $\rho=0.927$.

For DJIA, the U-shape of MEM-general $\sigma_{4}$ appears around the range of $0.81 \leq \lambda \leq 0.88$. The range for MEMgeneral $\sigma_{R V}$ is around $0.48 \leq \lambda \leq 0.59$. Their local min-
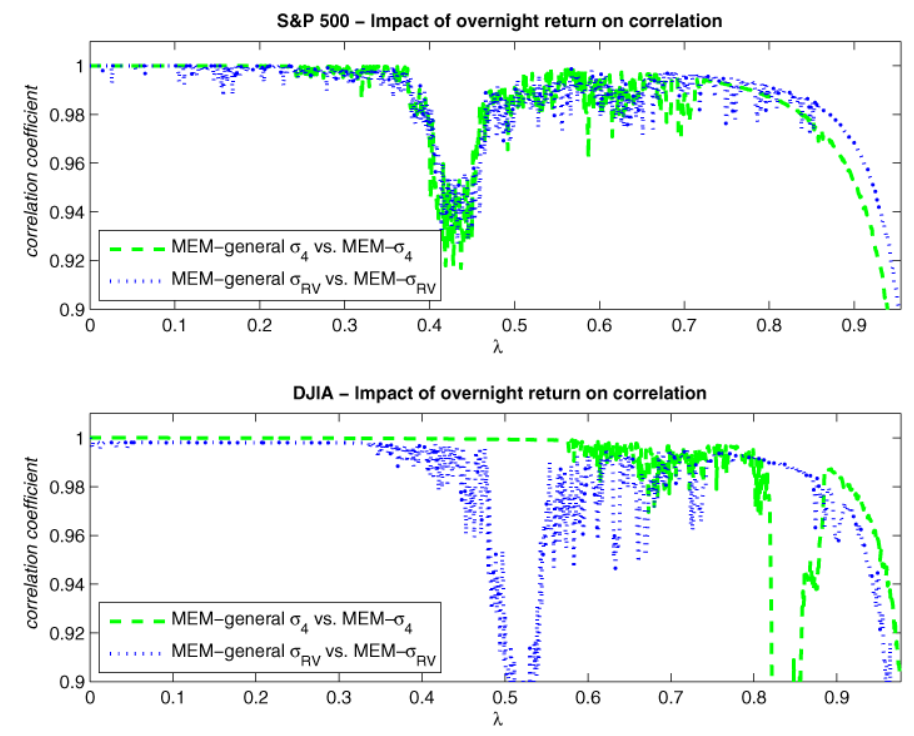

Figure 7. Correlation coefficient under the generalized weighted approach $(\rho \geq 0.9)$.

ima are at $\lambda=0.835$ with $\rho=0.765$ and $\lambda=0.517$ with $\rho=0.845$ respectively. The shift of the U-shape can be explained by the difference of the magnitudes between the $\hat{\sigma}_{4}^{2}$ and $\hat{\sigma}_{N W}^{2}$.

To summarize, the appearance of a U-shape portion in every curve demonstrates that the inclusion of overnight information can influence the characteristics of MEM volatility. However, the influence is not monotonic as an increase of overnight proportion does not necessarily produce a larger change in correlation.

\subsection{Incremental information content of model-based volatility}

Observing that overnight information gives noticeable impact in specific ranges, it is interesting to know whether the impact contains useful information or not. We assess the incremental information content by treating the MEM predicted conditional volatilities as exogenous variables to predict the market-based volatility indexes (VIX and VXD). For the simplicity, simple linear AR(1) OLS regression models are used in this study. The base model defined in equation 17 is used as the baseline for comparison purpose. MEM volatilities which help to increase the adjusted R-square and pass the join zero coefficient test with $95 \%$ confidence (indication of the coefficients are non-zeros with $95 \%$ confidence) are considered to contain incremental information content in this study. The results of adjusted R-square and F statistics are plotted in Figures 8, 9 and 10.

For both VIX and VXD regressions, models with combination $\lambda=0$ are checked and none of them can produce a regression result with larger adjusted R-square than the base model and pass the $\mathrm{F}$ test at the same time. Therefore, we regard that the MEM predicted volatilities without 

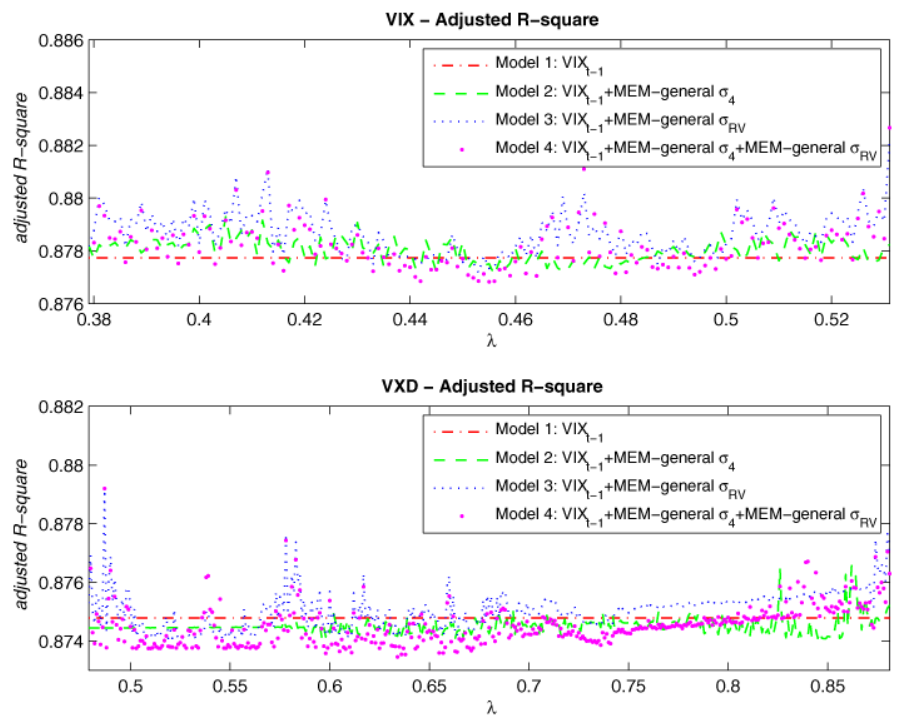

Figure 8. Adjusted R-square among the regression models.
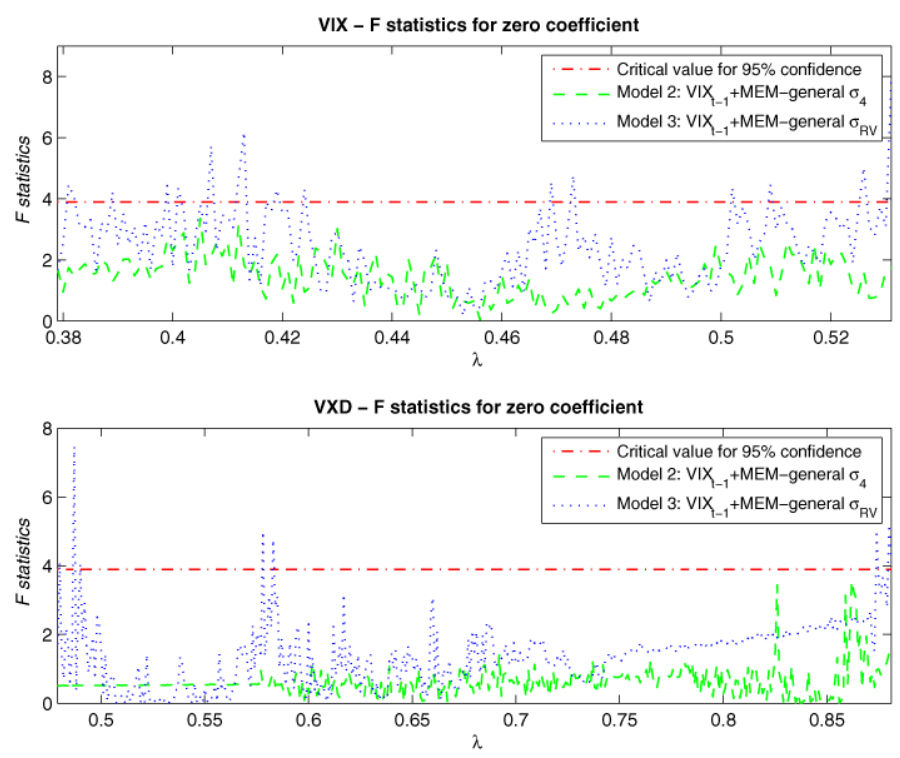

Figure 9. F statistics for testing zero coefficient.

overnight information do not contain incremental information for market-based indexes.

On the other hand, there are cases that give better R-square and pass the $\mathrm{F}$ test when overnight information is embedded. Table 3 lists the combinations that contain incremental information for the regression. The results for VIX and VXD regressions are consistent. The inclusion of MemRange ${ }_{t}$ cannot improve the prediction whereas $\mathrm{MemHighFeq}_{t}$ can give favorable results for some combinations. In additional, the information content of MemRange ${ }_{t}$ is not complementary to MemHighFreq $q_{t}$ as models with the use of both range-based and high-frequency information are always not as good as models that use $\mathrm{MemHighreq}_{t}$ alone.
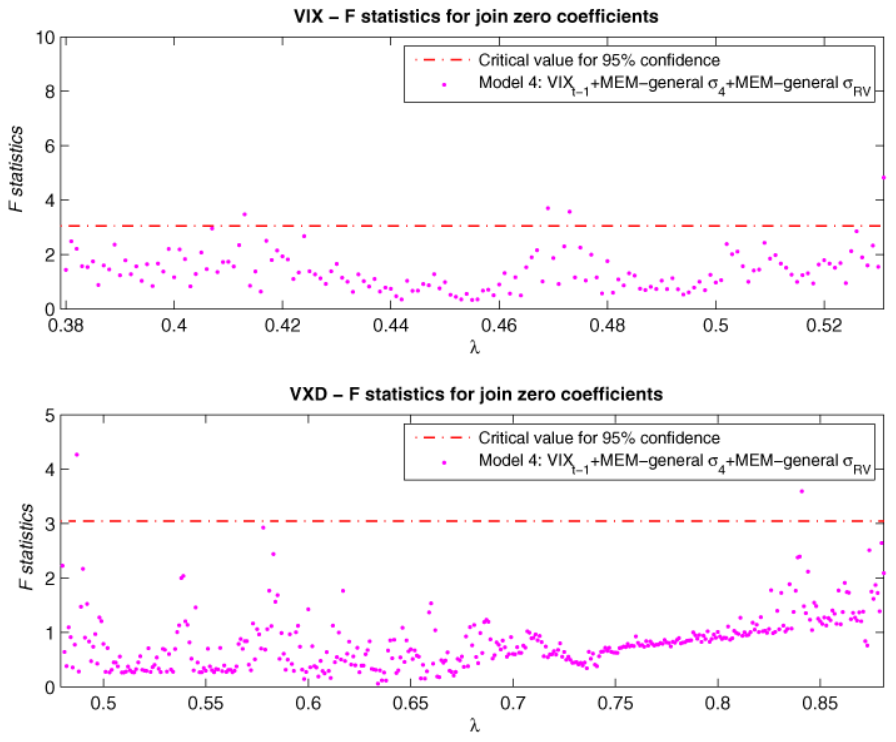

Figure 10. F statistics for testing join zero coefficients.

Table 3. Combinations $(\lambda)$ that give larger adjusted $R$-square than the base model and with $F$ statistics larger than $95 \%$ confidence critical value

\begin{tabular}{ccc}
\hline \multicolumn{3}{c}{ VIX regression } \\
\hline Model 2 & Model 3 & Model 4 \\
\hline & 0.381 & 0.413 \\
& 0.382 & 0.469 \\
& 0.389 & 0.473 \\
& 0.399 & \\
& 0.401 & \\
& 0.407 & \\
Nil & 0.412 & \\
& 0.413 & \\
& 0.419 & \\
& 0.424 & \\
& 0.469 & \\
& 0.473 & \\
& 0.502 & \\
& 0.509 & \\
& 0.526 & \\
\hline \hline Model 2 & VXD regression & \\
\hline \multicolumn{3}{c}{ Model 3 } \\
& 0.480 & \\
& 0.487 & \\
& 0.490 & \\
Nil & 0.578 & \\
& 0.583 & \\
& 0.874 & \\
& 0.880 & \\
& 0.881 & \\
& 0.885 & \\
\hline
\end{tabular}

Our empirical results exemplify that overnight information can improve the prediction of the CBOE volatility indexes under specific combinations. 


\section{CONCLUSION}

The impact of overnight return on Engle's Multiplicative Error Model (MEM) is investigated in this study. Under minimum-variance situations, overnight return has nearly no impact on models based on either Garman's or Hansen's whole-day-based estimators for both S\&P 500 and DJIA data. When the minimum-variance conditions are relaxed, our general formulations demonstrate U-shape patterns in the correlation graphs among the predicted conditional volatilities with and without overnight information. However, the influence is not monotonic as an increase of overnight proportion does not necessary produce a larger change in correlation.

On the other hand, our empirical results show that the inclusion of overnight information can improve the prediction of market-based volatility indexes, VIX and VXD, under specific combinations. We demonstrate MEM volatilities resulted from linearly combined overnight variance and high frequency realized variance can help to improve the prediction of CBOE indexes under a simple linear $\mathrm{AR}(1)$ OLS regression. More sophisticated regression models may further exploit the hidden value from overnight information. The above findings contradict the common perception that overnight return does not contain useful information for volatility prediction.

\section{Received 11 December 2007}

\section{REFERENCES}

[1] Andersen, T. G., Bollerslev, T., Christoffersen, P. F., and Diebold, F. X. (Jul 2006). Volatility and correlation forecasting, Chapter 15 of Handbook of economic forecasting (North-Holland), 777-878.

[2] Bandi, F. M. and Russell, J. R. (2004). Microstructure noise, realized volatility, and optimal sampling. Econometric Society 2004 Latin American Meetings. Santiago, Chile.

[3] Blair, Bevan J., Poon, Ser-Huang, and Taylor, Stephen J. (2000). Forecasting S\&P 100 Volatility: The Incremental Information Content of Implied Volatilities and High Frequency Index Returns. Accounting and Finance Working Paper No. 99/014. Lancaster University Management School.

[4] Bollerslev, T. (Apr 1986). Generalized Autoregressive Conditional Heteroskedasticity. Journal of Econometrics 31 307-327. MR0853051

[5] Cipollini, Fabrizio, Engle, Robert F., and Gallo, Giampiero M. (2007). A Model for Multivariate Non-negative Valued Processes in Financial Econometrics. Econometrics Working Papers Archive. Universita' degli Studi di Firenze, Dipartimento di Statistica "G. Parenti".

[6] Engle, R. (Fall 2001). GARCH 101: The Use of ARCH/GARCH Models in Applied Econometrics. Journal of Economic Perspectives 15 157-168.

[7] Engle, R. (2002). New Frontiers for ARCH models. Journal of Applied Econometrics 17 425-446.

[8] Engle, R. F. and Gallo, G. M. (2006). A multiple indicators model for volatility using intra-daily data. Journal of Econometrics 131 3-27. MR2275994
[9] Gallo, Giampiero M. (2001). Modelling the Impact of Overnight Surprises on Intra-Daily Volatility. Australian Economic Papers 40(4), Blackwell Publishing, 567-80.

[10] Garman, M. B. and Klass, M. J. (Jan 1980). On the Estimation of Security Price Volatilities from Historical Data. Journal of Business 53 67-78.

[11] Hansen, P. R. and Lunde, A. (2003). An Optimal and Unbiased Measure of Realized Variance based on Intermittent Highfrequency Data. CIREQCIRANO Conference, Montreal.

[12] Hansen, P. R. and Lunde, A. (Oct 2005). A Realized Variance for the Whole Day Based on Intermittent High-Frequency Data. Journal of Financial Econometrics 3 525-554.

[13] Hansen, P. R. and Lunde, A. (2005). A forecast comparison of volatility models: does anything beat a $\operatorname{GARCH}(1,1)$ ? Journal of Applied Econometrics 20 873-889. MR2223415

[14] Lanne, MarkKu (2006). A Mixture Multiplicative Error Model for Realized Volatility. Journal of Financial Econometrics 4(4) 594-616.

[15] Lam, K. P. and NG, H. S. (Jun 2006). Intra-daily information of range-based volatility for MEM-GARCH. International Conference on Time Series Econometrics, Finance, and Risk (TSEFAR), Perth.

[16] Lee, Sang-Won and Hansen, Bruce E. (1994). Asymptotic Theory for the GARCH $(1,1)$ Quasi-Maximum Likelihood Estimator. Econometric Theory 10 29-52. MR1279689

[17] Newey, W. K. and West, K. D. (May 1987). A Simple, Positive Semi-definite, Heteroskedasticity and Autocorrelation Consistent Covariance Matrix. Econometrica 55 703-708. MR0890864

[18] Oomen, Roel C. A. (2002). Modelling realized variance when returns are serially correlated. Warwick Business School, Mimeo.

[19] Parkinson, M. (Jan 1980). The Extreme Value Method for Estimating the Variance of the Rate of Return. Journal of Business 53 61-65.

[20] Poon, Ser-Huang and Granger, Clive W. J. (Jun 2003). Forecasting Volatility in Financial Markets: A Review. Journal of Economic Literature 41 478-539.

[21] Rogers, L. C. G. and Satchell, S. E. (Nov 1991). Estimating Variance From High, Low and Closing Prices. The Annals of Applied Probability 1 504-512. MR1129771

[22] Yang, D. and Zhang, Q. (Jul 2000). Drift-Independent Volatility Estimation Based on High, Low, Open, and Close Prices. Journal of Business 73 477-491.

[23] Zhang, J. and Shu, J. (2006). Testing Range Estimators of Historical Volatility. Journal of Futures Markets 26 297-313.

[24] Chicago Board Options Exchange (2003). Revised, more robust methodology for the VIX Index.

[25] Morgan/Reuters, J.P. (1996). Part II: Statistics of financial market returns. RiskMetrics technical document (Fourth Edition, New York).

C. F. Chu

Department of Systems Engineering and Engineering

Management

The Chinese University of Hong Kong

Shatin, N.T., Hong Kong

E-mail address: cf chu@se.cuhk.edu.hk

K. P. Lam

Department of Systems Engineering and Engineering

Management

The Chinese University of Hong Kong

Shatin, N.T., Hong Kong

E-mail address: kplam@se.cuhk.edu.hk 